\title{
Research on developing a measurement standard for insulation parameters on-line monitoring device
}

\author{
Jiliang Fu \& Ying Huang \& Xiwen Chen \& Zijuan Guo \& Xueqin Yu \\ China Electric Power Research Institute, Wuhan, China \\ $\mathrm{Xin} \mathrm{Lu}$ \\ Tian Jin Electric Power Company Electric Power Research Institute, Tianjin, China \\ Qihua Song \\ Zhe Jiang Electric Power Company Electric Power Research Institute, Hangzhou, China
}

\begin{abstract}
This paper is mainly to develop a measurement standard for insulation parameters on-line monitoring device. The paper achieves measurement standards for the Voltage, current, capacitance and dielectric loss data of insulation parameter monitoring device by voltage, current sync source generated by DDS technology; precision voltage source, constant current source module composed by op amp, voltage transformers and current transformers Group; Capacitive analog reference voltage and leakage current equipment. After being calibrated by the national legal metrology bodies, the metering standard technical indicators have reached the design requirements.
\end{abstract}

KEYWORD: Relative dielectric loss, Energized test, Measurement standards

\section{PREFACE}

Dielectric loss test has always been an important content of power-line system in online monitoring test. By measurement results of dielectric loss factor and capacitance test, combined with historical data and earlier data to determine insulation condition of the device. Insulation parameter online monitoring device is a device based on absolute or relative measurement method to measure the capacitive device dielectric loss factor and capacity (Fu, Wei-ping. 2006, Li, Xuan. et al. 2013, Zhu, De-heng. et al. 2009). Absolute measurements is a method that calculates the detected device's dielectric loss factor and capacitance according to the bus PT secondary voltage and detected device's current's ratio between dielectric loss factor and capacitance. Since the insulating parameter monitoring device belong to on-line monitoring instrument, using the relative measurement method foremost avoids the impact on PT angle deviation, secondly overcomes the uncertainty factor caused by ambient temperature, the amplitude of the voltage frequency fluctuations and load capacity changes. So the application of Insulation parameter monitoring devices is more widely used in the industry.

Currently, there is a variety of insulation parameter monitoring device models, but measuring quality is uneven. If in actual forth putting, the measurement results are not accurate, which may leads to false condition of equipment insulation, results in unnecessary losses. Therefore, to verify the insulation parameter monitoring device is necessary.
Projects funded: Science and technology project funding of State Grid Corporation of China.

At home and abroad research on online insulation monitoring device parameter measurement standards devices is a bit less, and the parameter index of existing measurement standard device is also very limited, urgent needs increasing demand. Therefore, the research and development of the online insulation monitoring device parameter measurement standards devices has become an important and need to be urgently overcome technical problem, the reliability evaluation method of the data also needs further study.

\section{THE THEORY OF ONLINE INSULATION PARAMETER MONITORING DEVICE}

Online insulation parameter monitoring device is used as measuring the capacitance-voltage transformer, coupling capacitor, transformer bushings, current transformers and capacitive device casing dielectric loss factor and capacitance. The theory of typical online insulation parameter monitoring device is shown in figure 1.

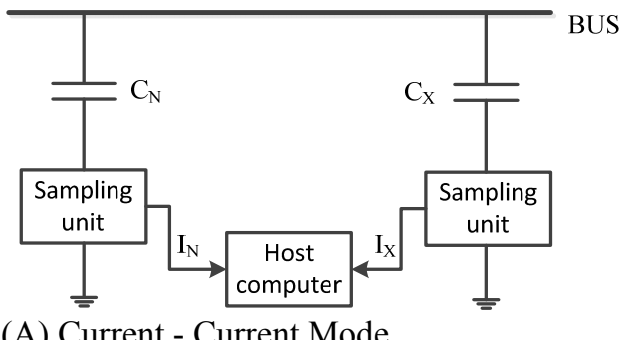

(A) Current - Current Mode 


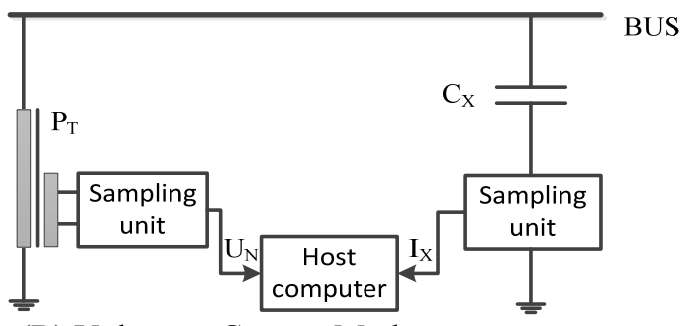

(B) Voltage - Current Mode

Fig.1 Insulation Parameters line monitoring device operating principle

There is two ways to measure online insulation parameter monitoring device: current - current type and voltage - current type. Current - Current - by means of test feedthrough current sensors to measure the same line in the same phase current, by comparison of the magnitude of the current ratio and phase difference to obtain the relative dielectric loss and capacitance ratio. Since the dielectric loss factor and capacitance of same type test sample are relatively close, and can be compared with historical measurement data, which can determine the insulation performance of the test according to the measurement results of the insulation parameter monitoring devices. Voltage current-feedthrough current transformer is by means of test feedthrough current sensor to measure the sample current, and to measure the same line PT secondary voltage of the sample by the voltage sensor, the current and voltage as well as PT ratio calculation to obtain sample's capacitance and relative dielectric loss factor, according to online insulation parameter monitoring device's measurement result well as historical data to judge the test sample.

For Current - Current type online insulation parameter monitoring device. The current signal amplitude through two capacitive equipment provided are I 1 and $I 2$, respectively, the phase are $\theta 1$ and $\theta 2$, the relative dielectric loss factor CX and capacitance DX can be obtained according to the following formula:

$$
\begin{aligned}
& D_{X}=\operatorname{tg}\left(\theta_{1}-\theta_{2}\right) \\
& C_{X}=I_{1} / I_{2}
\end{aligned}
$$

For voltage - current type online insulation parameter monitoring device. The current signal through capacitive equipment is $\mathrm{I}$, the voltage signal is $\mathrm{U}$, respectively, the phase are $\theta 1$ and $\theta 2$, the relative dielectric loss factor CX and capacitance DX can be obtained according to the following formula:

$D_{X}=\operatorname{tg}\left(\theta_{1}-\theta_{2}\right)$

$C_{X}=I / \omega U$

\section{THE MAIN FUNCTIONS OF A STANDARD MEASUREMENT DEVICE PARAMETERS}

The design parameters of measurement standard device are:
Current: output range $0.1 \mathrm{~mA} \sim 1 \mathrm{~A}$, the maximum permissible error $\pm(0.2 \%$ reading $+5 \mathrm{uA})$;

Voltage: output range $20 \mathrm{~V} \sim 200 \mathrm{~V}$, the maximum permissible error of $\pm 0.2 \%$ of reading;

Dielectric loss factor: Output range 0 to 1 , the maximum permissible error $\pm(0.2 \%$ reading +0.0005$)$;

Capacity: Output range of $30 \mathrm{pF} \sim 0.3 \mathrm{uF}$, the maximum permissible error $\pm(0.2 \%$ reading $+0.5 \mathrm{pF})$.

\section{THE DESIGN PRINCIPLE OF}

\section{MEASUREMENT STANDARDS APPARATUS}

Since there is two ways to measure online insulation parameter monitoring device: current - current type and voltage - current, the appropriate standards apparatus must also be able to provide two calibration mode.

The block diagram of online insulation parameter monitoring device measurement standards apparatus is shown in Figure 2.

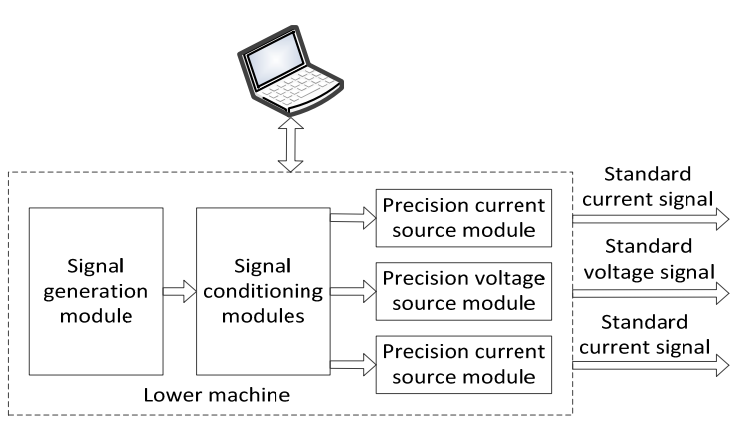

Fig.2 online insulation parameter monitoring device's Measurement Standard Device Block Diagram

For measuring principle of online insulation parameter monitoring devices, using design way of standard source, based on DDS devices to generate phase and standard amplitude adjustable signal, and by signal adjustment block and precision voltage amplification module or current amplification module and so on to design standard voltage source one and standard current source two, the amplitude and phase of the voltage signal and current signal source can be adjusted.

\section{INTRODUCTION OF MEASUREMENT STANDARD DEVICE CORE MODULE}

\subsection{Source module}

For the functional design of the measurement standard device, first need to generate a plurality of substantially sinusoidal signal unit, the sinusoidal signal unit are used to excitation rear constant current source circuit and a constant voltage source circuit and generates corresponding standard reference voltage, standard reference current and standard current. These sinusoidal signal units requirements should be 
met: amplitude adjusted, the amplitude resolution is 14 bits; frequency is stable and adjustable; sinusoidal is highly smooth, almost no ladder. Wherein the requirements of amplitude stable and adjustable is easier to achieve by the conventional design ideas, such as use an ordinary microcontroller according to sine table queries method and so on operating on the highresolution ADC chip can achieve, but this method isn't beneficial to sinusoidal signal frequency's stable and adjustable, especially to the signal's curve smoothing, the main reason is: the limited existing SCM's processors and so on and ADC chip operating speed is limited, in the control of SCM and so on processor use ADC chip generating sinusoidal signal is only reach one point every tens of micros, this way, generated sine wave has obvious "ladder", that is not a smooth curve. In order to achieve a smoothing effect, they often need to add a filter circuit in the subsequent conditioning circuits, which filter circuit in turn leads to input "ladder" sine wave's amplitude attenuation and phase shift, and the impact of the amplitude attenuation and phase shift by the filter circuit devices and input signal frequency lead into greater uncertainty, which is harmful to improve the accuracy of measurement standard device's reference voltage and current's amplitude, frequency, phase the three key parameters .

Primary in order to obtain stable and adjustable amplitude and frequency, and smooth sine curve signal unit, the paper choses DDS (direct digital synthesis) chip to generate various views of original excitation signal that is differential sinusoidal signal. Direct digital synthesis technology is mainly used in the field of high-frequency signals, less used in the field of electrical testing. Its main feature is based on direct digital synthesis theory, which comes up with a method to generate rapidly changing frequency sinusoidal signal (or arbitrary waveform cycle), use this technology in this issue can easily generate sinusoidal signal which frequency is stable and adjustable amplitude is stable and adjustable、 phase is stable and adjustable and height of the curve is smooth and stable. This paper uses DDS chip AD9951. Based on the chip can achieve: the frequency of the sinusoidal signal is $50 \mathrm{~Hz}$ and very stable; sinusoidal signal amplitude resolution reach 14 bits, has met the needs of the subject; more importantly, sinusoidal curve is still discretization, but the curve amplitude refresh interval is less than lus, very smooth, no longer see "ladder" through ordinary oscilloscope, the " curve is highly smooth" completely avoidable uses follow-up filter circuit, which can greatly reduce the amplitude and phase negative effects which filter circuit caused .

\subsection{Precision voltage source module}

The circuit schematic of precision constant voltage source diagram is shown in Figure 3, is composed of the input and output ports, a voltage amplifying circuit, a DC clamp circuit, a high frequency filter circuit. Operational amplifier U1, resistors R4, resistor $\mathrm{R} 5$, transformers $\mathrm{T} 1$ and $\mathrm{T} 2$ compose of $\mathrm{AC}$ voltage negative feedback circuit. In AC-working band: the Op amp's output voltage capability is $U_{m}$, the ratio of the input and output voltage of transformer $\mathrm{T} 1$ is $\mathrm{N}$, so the amplifier output voltage capability is $\mathrm{NU}_{\mathrm{m}}$; he ratio of the input and output voltage of voltage transformer is $\mathrm{K}$, the equation between output voltage $\mathrm{U}_{\mathrm{o}}$ and input voltage $U_{i}$ of the amplifier is: $\mathrm{U}_{\mathrm{o}}=\mathrm{U}_{\mathrm{i}} / \mathrm{K}$.

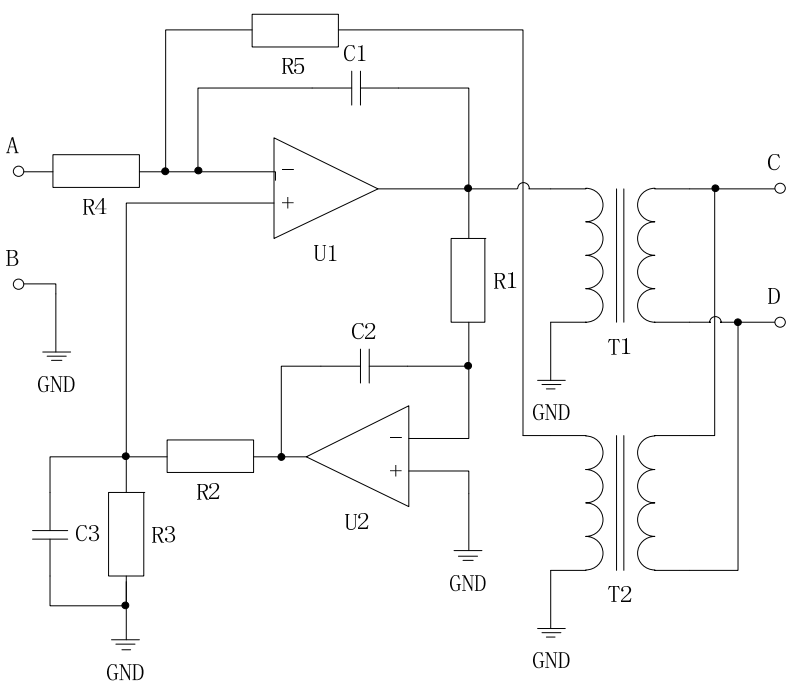

Fig. 3 The circuit schematic of precision constant voltage source diagram

Because the transformer T1, voltage transformer T2 are both nonlinear elements, DC signals can't pass and high-frequency signal will generate additional phase shift, and therefore needs to add the current feedback and high frequency feedback loop, to assure the normal operation of the amplifier circuit. So add the DC clamp circuit and high-frequency filter circuit. DC clamp circuit is composed of the integrator circuit and a low-pass filter circuit, so that the operational amplifier U1 output terminal DC level is clamped to the ground point, while the working frequency AC signal is hardly affected. High-frequency filter circuit make the high-frequency component through a capacitor $\mathrm{C} 1$ feedback to inverting input terminal of operational amplifier U1, to avoid the amplifier circuit oscillation.

Except step-up transformer output circuit is in high voltage state, the other part of the circuit elements including power supplies are all working in low voltage, the utilization of power is efficient, the cost is low; the output voltage changes with the input voltage signal in real time, the output voltage waveform and phase are decided by precision voltage transformer, accuracy is high. Voltage output circuit is isolated with other circuit electrical elements, can effectively 
protect the circuit elements, the loads have no problem of having the common ground.

\subsection{Precision current source module}

The circuit schematic of precision constant current source diagram is shown in Figure 4, is composed of input and output ports, a current amplifying circuit, a DC clamp circuit, a high frequency filter circuit. Operational amplifier U1, transformers $\mathrm{T} 1$ and $\mathrm{T} 2 \mathrm{com}-$ pose of AC current negative feedback circuit. In ACworking band: the Op amp's output current capability is $\mathrm{I}_{\mathrm{m}}$, the ratio of the output and input current of transformer $\mathrm{T} 1$ is $\mathrm{N}$, so the amplifier output current capability is $\mathrm{NI}$; the ratio of the input and input current of current transformer is $\mathrm{K}$, the equation between output current $\mathrm{I}_{\mathrm{o}}$ and input current $\mathrm{I}_{\mathrm{i}}$ of the amplifier is: $\mathrm{I}_{\mathrm{o}}=\mathrm{I}_{\mathrm{i}} / \mathrm{K}$.

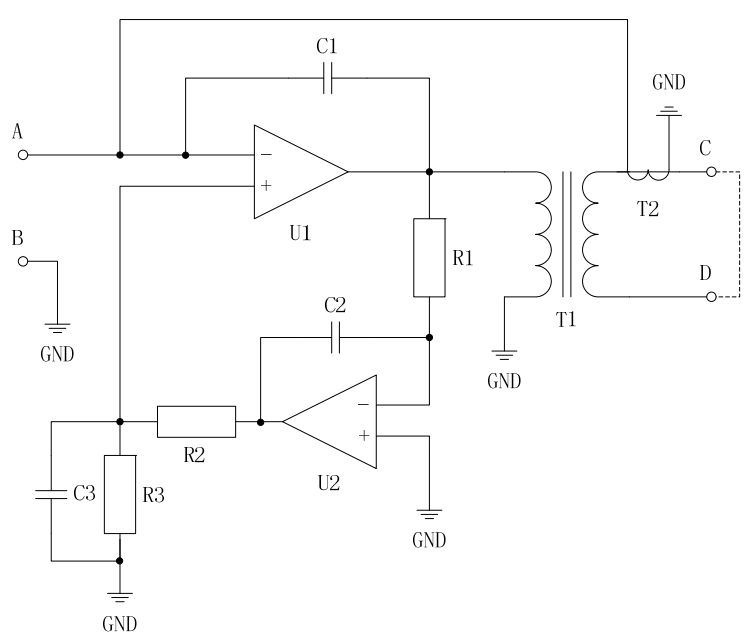

Fig.4 The circuit schematic of precision constant current source diagram

Because the transformer T1, voltage transformer $\mathrm{T} 2$ are both nonlinear elements, DC signals can't pass and high-frequency signal will generate additional phase shift, and therefore needs to add the current feedback and high frequency feedback loop, to assure the normal operation of the amplifier circuit. So add the DC clamp circuit and high-frequency filter circuit. DC clamp circuit is composed of the integrator circuit and a low-pass filter circuit, so that the operational amplifier U1 output terminal DC level is clamped to the ground point, while the working frequency $\mathrm{AC}$ signal is hardly affected. High-frequency filter circuit make the high-frequency component through a capacitor $\mathrm{C} 1$ feedback to inverting input terminal of operational amplifier U1, to avoid the amplifier circuit oscillation.

Except step-up transformer output circuit is in high current state, the other part of the circuit elements including power supplies are all working in low current, the utilization of power is efficient, the cost is low; the output current changes with the input current signal in real time, the output current waveform and phase are decided by precision current transformer, accuracy is high. Current output circuit is isolated with other circuit electrical elements, can effectively protect the circuit elements, the loads have no problem of having the common ground.

\section{EXPERIMENTAL DATA}

\subsection{The calibration method}

Diagram of measurement standard device Calibration test circuit is shown in Figure 5.

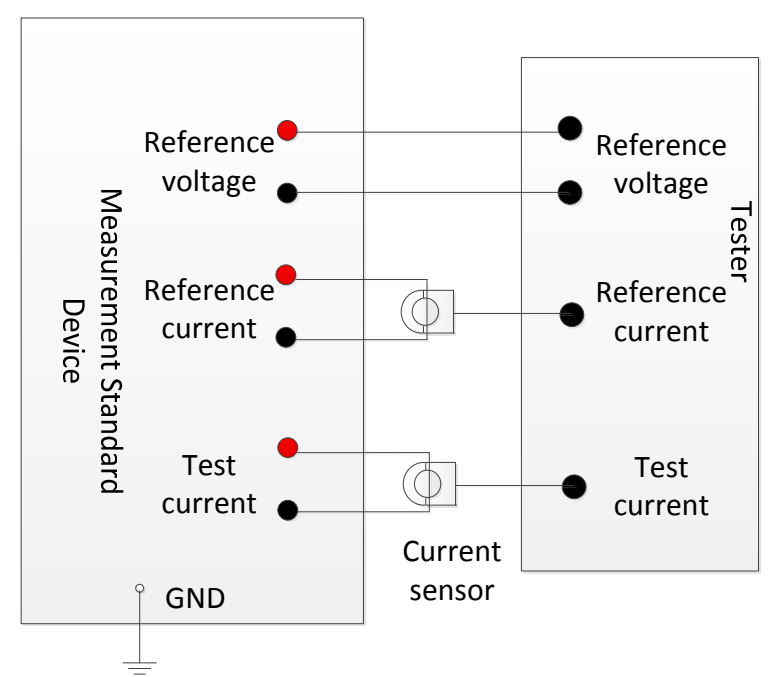

Fig.5 Diagram of measurement standard device Calibration test circuit

When test personnel using this calibration test to carry out online insulation parameter monitoring device calibration, firstly Connect test line, monitoring voltmeter and a corresponding voltage and current sensor s according to Figure 5, connect the ground wire power cable and so on in the back panel, power cable, open the lower machine power switch and PC software interface. Before the test, first set the reference voltage, reference current, test current, dielectric loss factor, capacity and so on other test points, and then click the start button. If the communication is not normal, it will prompt communication error. If the communication is normal, the $\mathrm{PC}$ will send set argument to the next crew, if started successfully, it will output reference voltage and current signals, start test, read the measurement results of the test insulation parameters online monitoring device, compare test insulation parameters online monitoring device dielectric loss factor, capacity and so on with the value of insulation parameters online monitoring device measurement standard device have set, you can get the deviation of current measurement point.

The standard measurement device can be traced back to power analyzers by Figure 6,to ensure accurate and reliable of standard measurement value. 


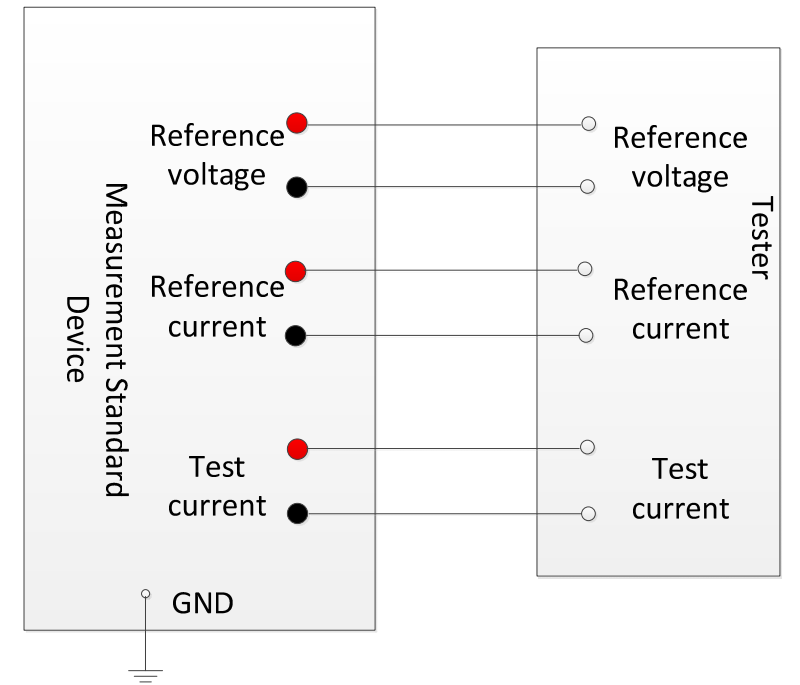

Fig.6 Traceability wiring diagram

\subsection{The calibration data}

Table 1 is example table about part of the calibration data of insulation parameters online monitoring device measurement standard device.

Table 1 Calibration data of measurement standard device

\begin{tabular}{cccc}
\hline \hline $\begin{array}{c}\text { Phase } \\
\text { standard } \\
\text { value }\left(^{\circ}\right)\end{array}$ & $\begin{array}{c}\text { D standard } \\
\text { value }\end{array}$ & D test & $\begin{array}{c}\text { Expanded uncer- } \\
\text { tainty } \\
\mathrm{U}(\mathrm{k}=2)\end{array}$ \\
\hline 89.933 & $0.117 \%$ & $0.10 \%$ & $7.3 \times 10-5$ \\
89.876 & $0.216 \%$ & $0.20 \%$ & $7.4 \times 10-5$ \\
89.706 & $0.513 \%$ & $0.50 \%$ & $7.6 \times 10-5$ \\
89.415 & $1.021 \%$ & $1.00 \%$ & $7.9 \times 10-5$ \\
88.844 & $2.018 \%$ & $2.00 \%$ & $8.6 \times 10-5$ \\
87.128 & $5.017 \%$ & $5.00 \%$ & $1.1 \times 10-4$ \\
84.277 & $10.022 \%$ & $10.00 \%$ & $1.5 \times 10-4$ \\
78.674 & $20.029 \%$ & $20.00 \%$ & $2.2 \times 10-4$ \\
63.423 & $50.026 \%$ & $50.00 \%$ & $4.3 \times 10-4$ \\
44.993 & $100.024 \%$ & $100.00 \%$ & $7.8 \times 10-4$ \\
\hline
\end{tabular}

As can be seen from the calibration data, insulation parameters online monitoring device measurement standard device meet the test requirements of insulation parameter monitoring devices.

\section{CONCLUSION}

Implemented based DDS insulation parameters online monitoring device measurement standard device standard source method, have been applied to calibration of insulation parameters online monitoring device, have ensured the effectiveness and accuracy of traceability chain, have played a positive role in promoting insulation parameter online monitoring apparatus to standardization, normalization .

\section{REFERENCES}

Fu, Wei-ping. 2006. Method on Field Living Test of $220 \mathrm{kV}$ Current Transformer [J], High Voltage Engineering, 32 (1): 111-113.

Li, Xuan. et al. 2013. Effect of CVT Additional Phase Error on On-Line Monitoring of Dielectric Loss Factor for Capacitive Equipment, Electrical Measurement \& Instrumentation, 50 (11): 10-13.

Zhu, De-heng. et al. 2009. Condition Monitoring and Fault Diagnosis Technology for Electrical Equipments [M]. Beijing: China Electric Power Press. 